\title{
Kernos
}

Revue internationale et pluridisciplinaire de religion grecque antique

17 | 2004

Varia

\section{Les dieux dansent chez Proclus}

\section{Evanghelos A. Moutsopoulos}

URL : http://journals.openedition.org/kernos/1410

DOI : $10.4000 /$ kernos. 1410

ISSN : 2034-7871

\section{Éditeur}

Centre international d'étude de la religion grecque antique

Édition imprimée

Date de publication : 1 janvier 2004

ISSN : 0776-3824

\section{Référence électronique}

Evanghelos A. Moutsopoulos, «Les dieux dansent chez Proclus », Kernos [En ligne], 17 | 2004, mis en ligne le 22 avril 2011, consulté le 01 mai 2019. URL : http://journals.openedition.org/kernos/1410 ; DOI : 10.4000/kernos. 1410 


\section{Les dieux dansent chez Proclus'}

Sous forme d'entités jouissant d'un statut de réalités symboliques, les divinités de la Grèce classique, oubliées par Plotin, sont, au contraire, omniprésentes dans la pensée de Proclus, commentateur néoplatonicien tardif, mais extrêmement original. Elles comblent les interstices et les vides entre les hypostases plotiniennes, au point qu'à une discontinuité de la hiérarchie ontologique, elles contribuent à substituer une quasi-continuité qui fait osciller le système proclusien entre dualisme et monisme. L'architecto-nique de ce système diffère donc radicalement de celle du système plotinien; ses éléments, dont les dieux grecs eux-mêmes, sont en mouvement perpétuel. Dans leur ensemble, les dieux grecs y jouent à la fois le rôle de moteurs et de mobiles. Mentionner ici leurs noms en détail - Cronos, Zeus, Héra, Athéna, Apollon, dieux de premier ordre; Sirènes, Moires, Muses, Grâces et autres divinités secondaires, à côté de puissances de troisième importance, ne saurait en aucun cas faire avancer notre enquête. Par conséquent, mieux vaut les envisager in abstracto dans un contexte fonctionnel généralisé.

Autour de l'Un, considéré comme Père, évoluent le chour des hénades' et des dieux supérieurs ${ }^{2}$ ainsi que d'autres puissances que Proclus qualifie d'anges et de démons ${ }^{3}$. La série des rondes se poursuit selon la hiérarchie ontologique. Le mouvement du ciel imite le mouvement des dieux supralunaires, lui-même imitation du mouvement des dieux supra-célestes", et engendre, à son tour, celui des dieux sublunaires", entraînés de la sorte dans un cercle

* Nous avons calqué le titre de cette étude sur celui du livre classique de J. CAzEnEuve, Les dieux dansent à Cibola, Paris, Gallimard, 1956.

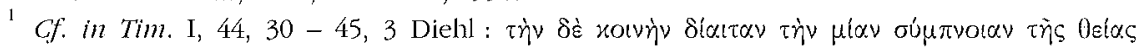

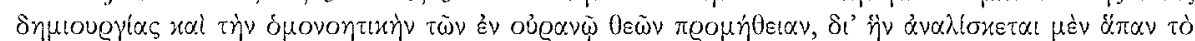

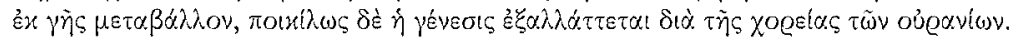

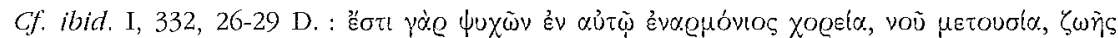

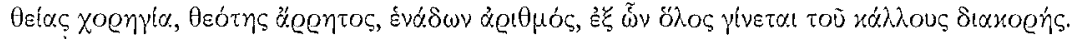

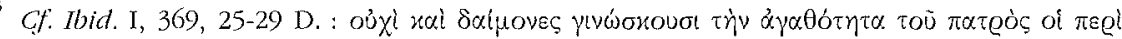

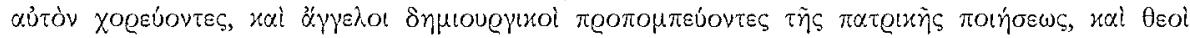

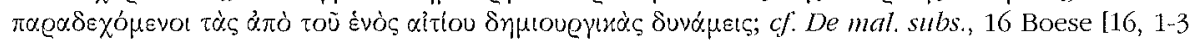
Isaacl.

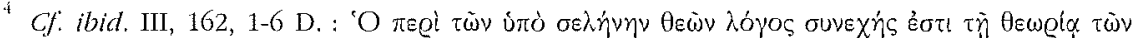

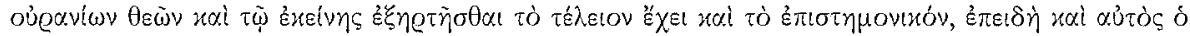

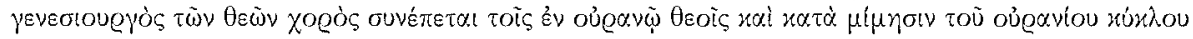

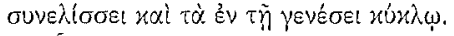

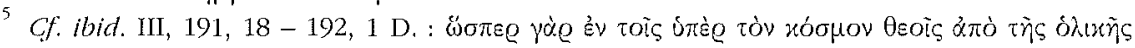

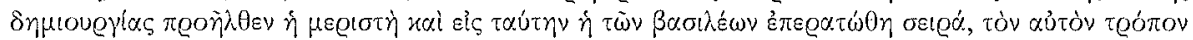

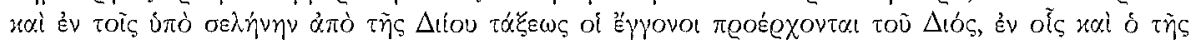

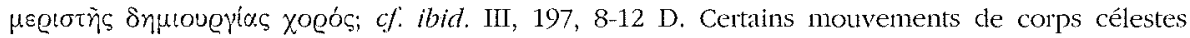


choreutique: entraînement répété à tous les niveaux de la hiérarchie cosmique, des âmes" et des êtres animés ${ }^{7}$. Les âmes particulières évoluent autour de l'Âme supérieure ${ }^{8}$ qui, elle, évolue autour de l'Intellect'. Il apparaît déjà que les chœurs des diverses puissances se mêlent aux chœurs des âmes. Or les choses se compliquent à plaisir au niveau cosmique. L'univers, en tant qu'ensemble structuré soumis à un mouvement continu, est sujet à une gravitation autour d'un centre ${ }^{10}$ qui est la Terre". Qui dit univers dit voûte céleste constituée de sphères concentriques ${ }^{12}$. Il semble cependant que, pour Proclus, ces sphères ne se meuvent pas en bloc, mais que chacune d'elles évolue indépendamment et selon (ávò $\lambda o ́ \gamma o v$ ) la ronde qu'accomplissent les astres dont elle est composée ${ }^{13}$. Autour de la Terre évoluent également toutes

semblent irrationnels, parce que désordonnés; ils peuvent néanmoins être expliqués en tant qu'apparents grâce au recours à la raison (Hy)p. astr., 4, 25-6,5 Manitius), surtout s'il s'agit du soleil et de la lune (ibid., 16, 9-12 Manitius). Or Proclus est un fervent supporteur des thèses de Ptolémée.

${ }^{6}$ Cf. ibid. III, 262, 21-26 D. Cf. Théol. plat. II, 3, 347 Portus [p. 16, 19-27 Saffrey-Westerink]; II, 18, 395 P. [p. 85, 1-3 S.-W.]; VI, 21, 401 P. [p. 94, 10-14 S.-W.]; VI, 22, 404 P. [p. 98, 25-27 S.W.].

Cf. in 7im. 222, 7-10 D.

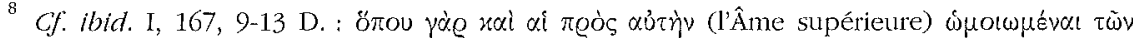

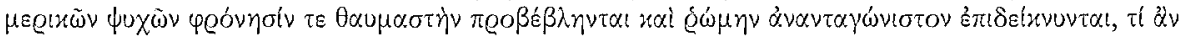

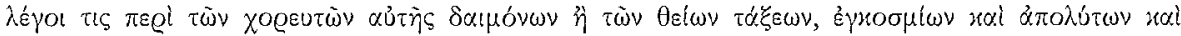

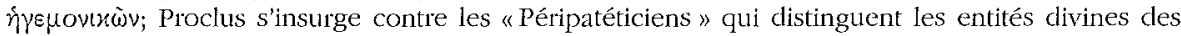
entités intelligibles. Il concède pourtant que l'ensemble du ciel sensible désire les intelligibles et donc gravite autour d'eux. Cf. in Pamen. 921, 18-24 C., qui note ici des contradictions. Ailleurs (Théol. plat. V, 3, 253-254 P. [p. 16, 24 S.-W.], Proclus mentionne des noeroi theoí.

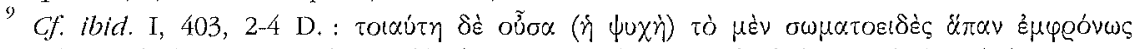

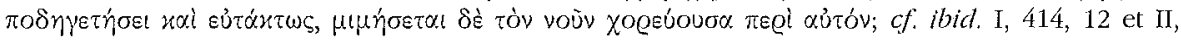

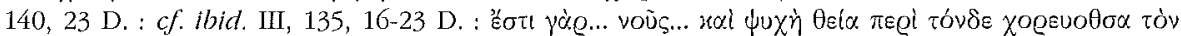

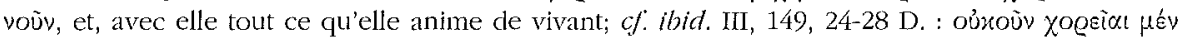

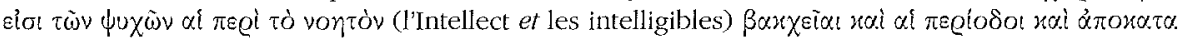

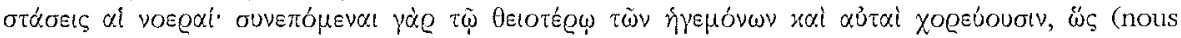

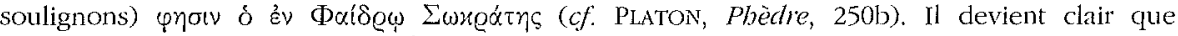
l'Intellect autour duquel gravite l'Âme évolue, de son côté, autour de I'Un. Cf. in Pamen. 1161,

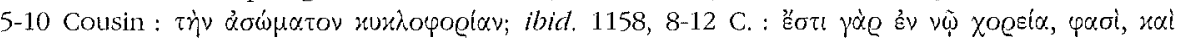

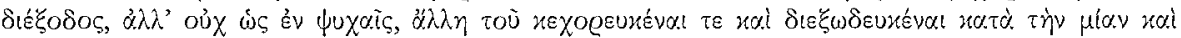

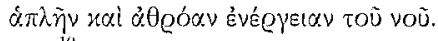

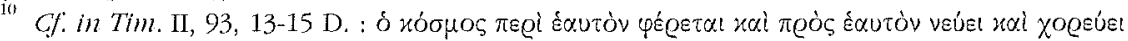

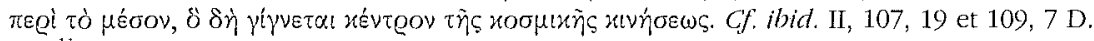

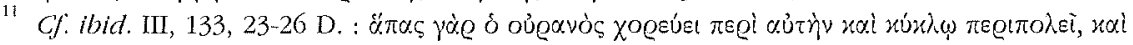

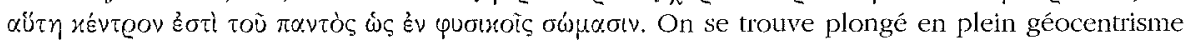
ptoléméen. L'origine, éventuellement platonicienne, de l'héliocentrisme aristarchéen semble avoir été définitivement oubliée. Cf. E. Moutsopoulos, "Sur l'origine philosophique possible du modèle de l'univers aristarchéen ", Diotima 12 (1984), p. 175-177. Cf. in Tim. III, 146, 4-5 D. : oủ

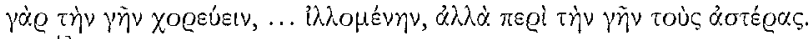

${ }_{12}$ Sur la tradition et le bien-fondé ou non de la théorie relative à l'harmonie des sphères, $c /$. E. Moutsopoulos, La musique dans l'ouvre de Platon, Paris, PUF, 1989², p. 375-383.

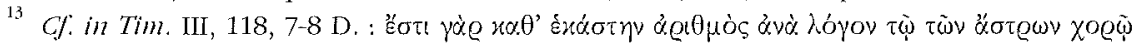

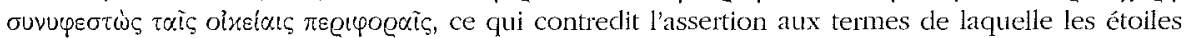
se meuvent d'un mouvement unique et uniforme; cf. in Tim. III, 146, 6-7 D. : Xogsúovol yòe (sc. 
sortes de puissances ${ }^{14}$, qui meublent l'univers proclusien ontologiquement dilaté, et à la ressemblance de ce qui se passe autour de l'Âme supérieure ${ }^{15}$.

C'est ici qu'intervient la ronde du temps ${ }^{16}$. En fait, le temps n'est pas un chour en soi, mais une structure réelle moyennant laquelle il est possible de saisir le mouvement du devenir par rapport à la stabilité de l'être et à l'éternitét ${ }^{17}$. En proposant une étymologie fantaisiste, du genre de celles avancées par Platon dans son Cratyle ${ }^{18}$, Proclus rapproche la nature du temps à la fois des natures de l'Intellect et de la création ${ }^{19}$. La danse du temps est un retour incessant $^{20}$. C'est pourquoi sa présence est tant mobile que continue ${ }^{21}$. Une révolution complète du temps permet de saisir le passé; une révolution

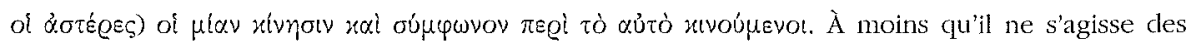
étoiles que comporte chacune des sphères.

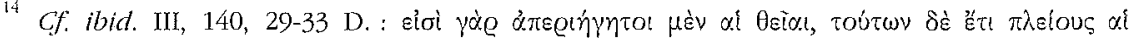

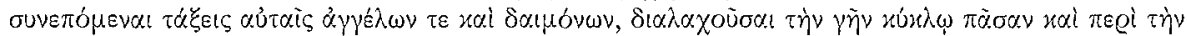

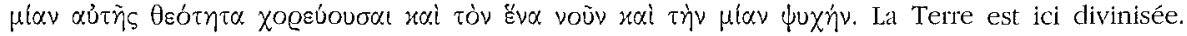
Dieux, démons, âmes et autres entités se comportent en danseurs qui gravitent autour du «dieu », fascinées qu'elles sont par le chant des Sirènes. Cf. in Crat., 88, 24-26 Pasquali.

${ }^{15}$ Cf. supra et la note 5.

${ }^{16}$ Sur la nature du temps, cf. E. MOUTSOpoulos, Structure, présence et fonctions du kairos chez Proclus, Athènes, Académie, 2003, p. 19-36 et 96-105.

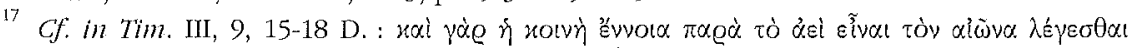

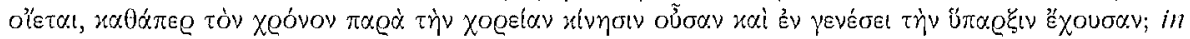

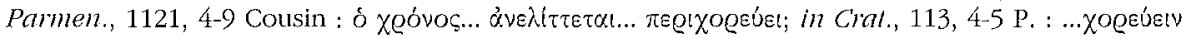

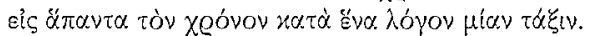

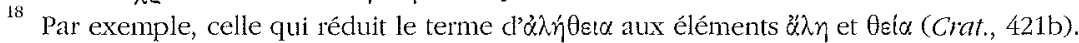

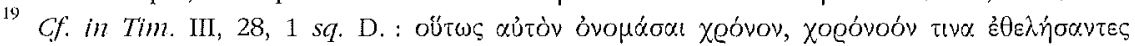

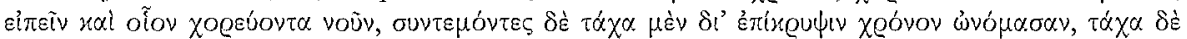

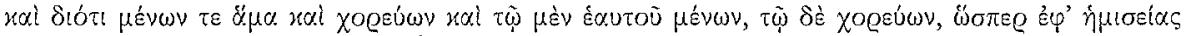

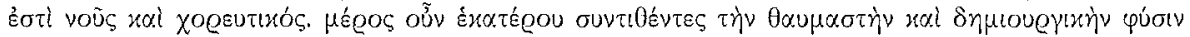

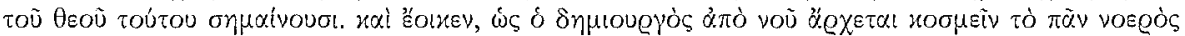

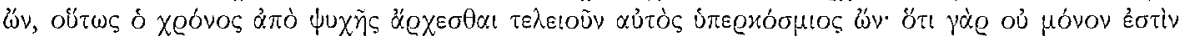

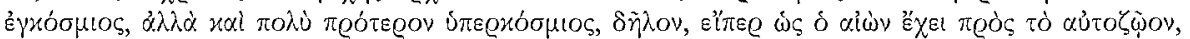

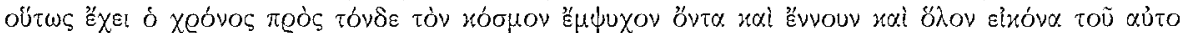

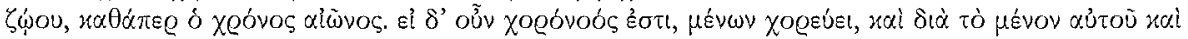

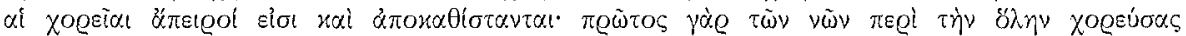

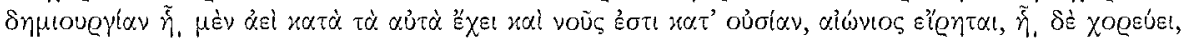

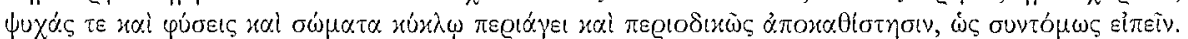

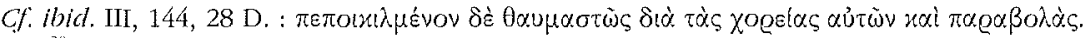

2i) Cf. ibid. III, 51, 5-7 D. : oủ $\lambda \eta \hat{\xi}$

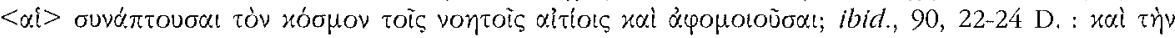

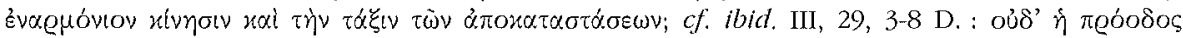

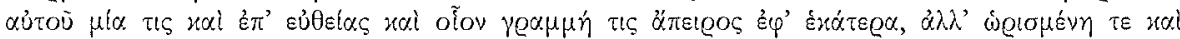

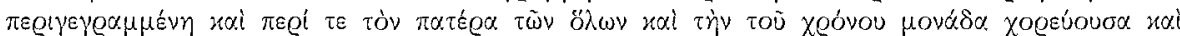

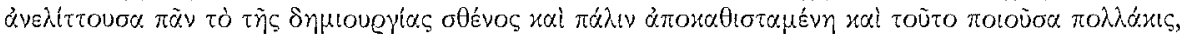

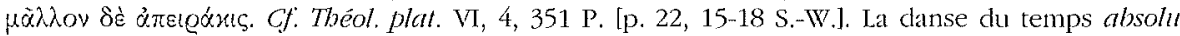
sert à mesurer les révolutions des âmes; cf. in Parmen., 1217, 16-21 C.

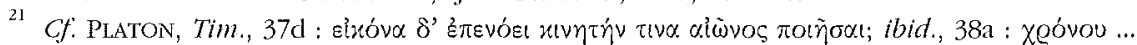

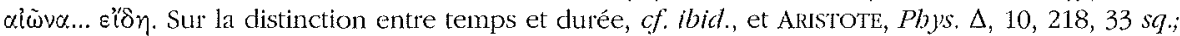

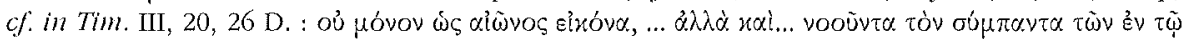

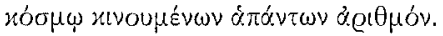


encore incomplète, le devenir ${ }^{22}$. Entre les deux se situe le présent qui, dans le cas du kairos, devient, entre autres, un présent vécul, par anticipation ou à retardement, selon le $\operatorname{cas}^{23}$. L'univers est, quant à lui, entièrement soumis à la révolution, indéfiniment répétée, du temps, produit d'une "création dans le temps », et donc spécialement créé avec elle ${ }^{24}$. Au cours de ses révolutions, et parallèlement aux sphères célestes, le temps marque le pas par le nombre, conformément au chœur des intelligences et des intelligibles ${ }^{25}$. C'est en dansant autour des intelligibles que les âmes sont en mesure de les contempler $^{26}$, et que les corps évoluant autour des âmes se rendent dignes d'en être imprégnés ${ }^{27}$. A son tour, la connaissance naît du mouvement circulaire de l'intelligence autour de l'être particulier qui en constitue l'objet ${ }^{28}$. De même que les idées, objets de contemplation, les vertus forment leur chœur cyclique en un lieu où il est agréable de résider ${ }^{29}$. Rien d'étonnant, dès lors, que les

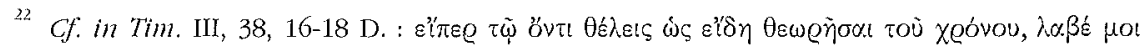

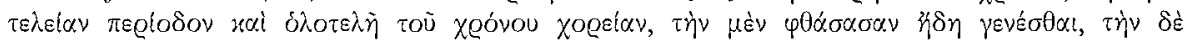

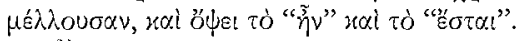

23 Cf. MOUTSOPOUlOS, o.c. (n. 16), p. 22-25.

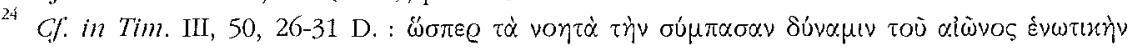

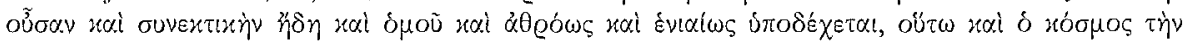

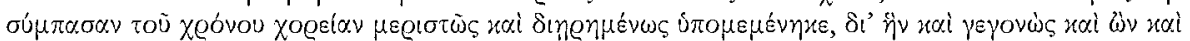

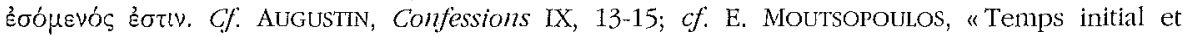
temps initiatique chez Augustin. Une interprétation intentionnaliste », Glormale di Metafisica 13 (1991, p. 231-242.

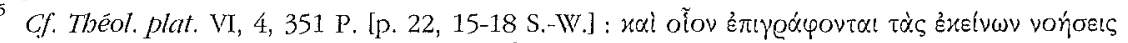

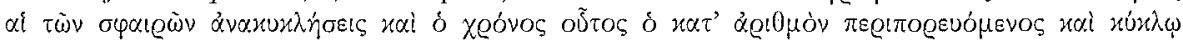

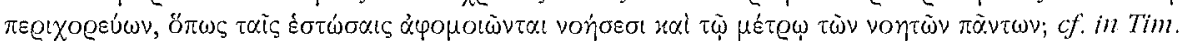

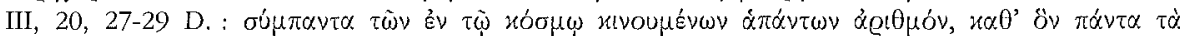

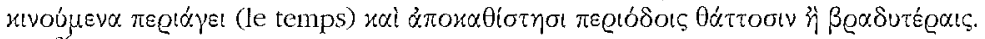

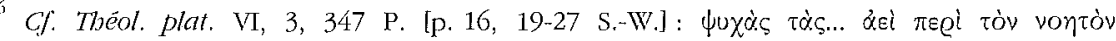

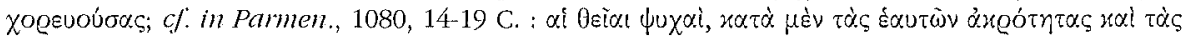

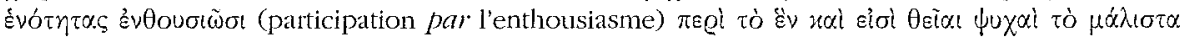

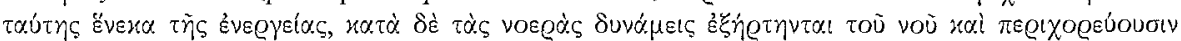

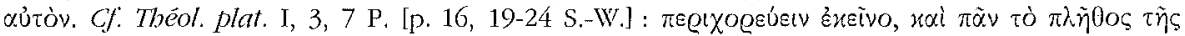

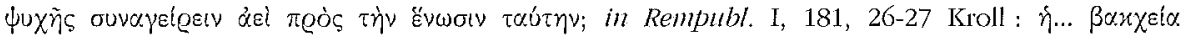

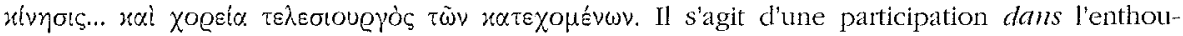
siasme; cf. ibid. VI, 18, 395 P. [p. 85, 1-3 S.-W.]; 21, 401 P. [p. 94, 10-14 S.-W.]; 22, 404 P. [p. 98,

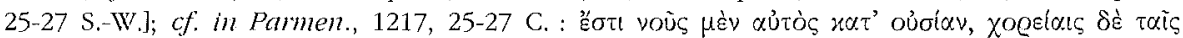

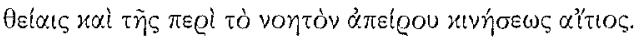

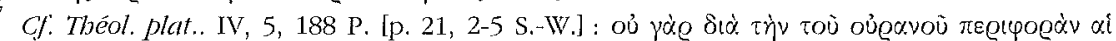

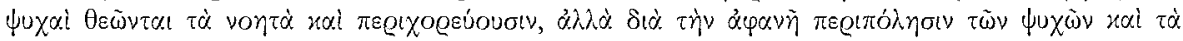

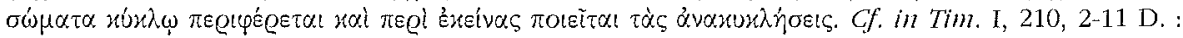

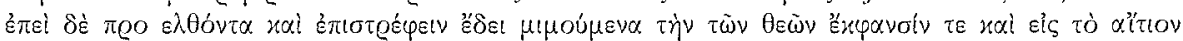

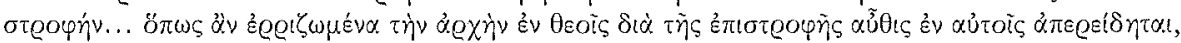

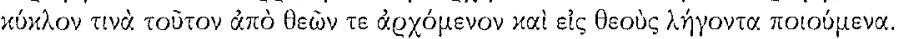

${ }^{28}$ Cf. Théol. plat. IV, 13, 200 P. [p. 43, 20-23 S.-W.] : (la connaissance)... éveQyei is

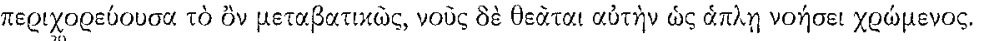

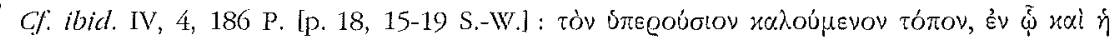

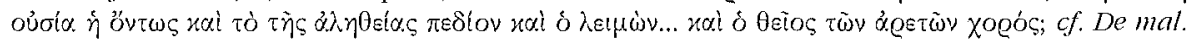

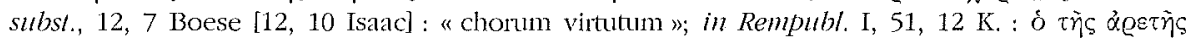
xogós. 
philosophes, Platon par exemple $e^{3 !}$, aient eu, eux aussi, un cercle de disciples enthousiastes qui les imitaient " en dansant autour d'eux ».

Des considérations qui précèdent il convient de tirer trois conclusions. Premièrement, mis à part la voûte céleste, avec ses sphères concentriques en rotation, qui, en fait, tourne non autour d'un axe quelconque, mais autour de la Terre même, tout l'univers des hypostases, excepté l'Un imperturbable, se trouve en mouvement. Ce mouvement est-il effectivement circulaire? La réponse à cette question sera à la fois affirmative et négative. D'une part, il est indéniable que ce qui se meut autour de l'Un suit une trajectoire circulaire; mais que ce qui se meut circulairement autour de ce qui déjà se meut circulairement ne saurait suivre une trajectoire circulaire simple. Deux hypothèses peuvent éventuellement être émises à ce propos: (a) s'il se meut sur le même plan que le mouvement circulaire du centre de son propre mouvement, ce dernier pourrait être cyclö̈de et lui permettre tantôt de s'en rapprocher, tantôt de s'en éloigner tant soit peu; (b) si, par contre, il se meut dans l'espace, son mouvement ne peut être qu'bélicoïdal ${ }^{31}$.

A partir de ces déductions, un schéma choral de cercles hiérarchisés, compliqué à loisir, pourrait être esquissé : autour de chaque choreute graviterait une multitude d'autres choreutes, et ainsi de suite ${ }^{32}$. Les divergences et inadéquations occasionnelles touchant les diverses entités et leur relations hiérarchiques, observées lors des confiontations et comparaisons entre passages parallèles, seraient, à tout prendre, naturelles dans une cuvre aussi vaste que celle du Diadoque. Il convient donc de passer résolument outre.

Deuxièmement, il y a lieu de se demander quelle est la véritable raison d'être de chacun de ces cercles. La réponse nous vient de Proclus lui-même quand il fait état du désir de chaque entité d'atteindre l'entité immédiatement supérieure et, finalement, de son "désir de l'Un »" C'est donc un désir qui le fait graviter autour de l'entité désignée ${ }^{34}$; un désir déjà programmé, sous forme

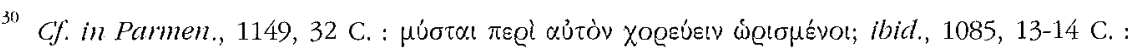

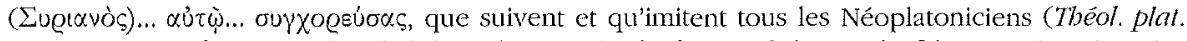

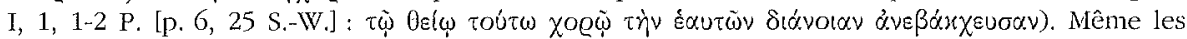
éléments du tissage s'entremêlent comme dans une danse (cf. in Crat., 21, $23-22,10 ; 113,3-8$ P.).

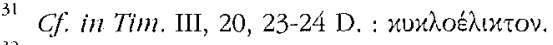

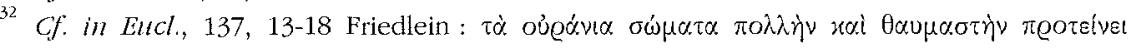

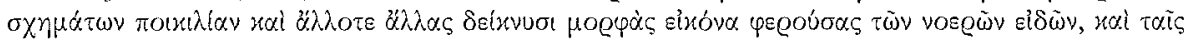

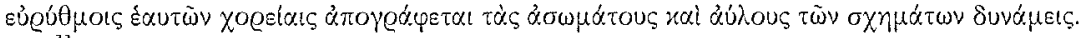

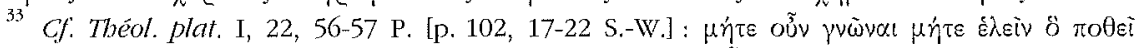

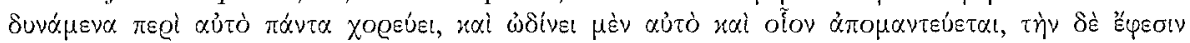

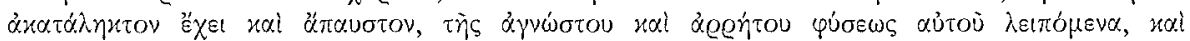

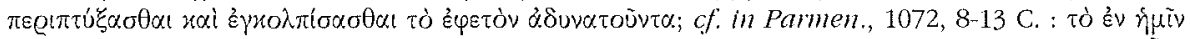

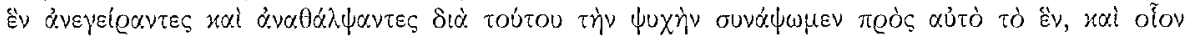

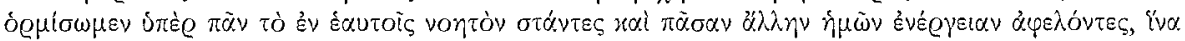

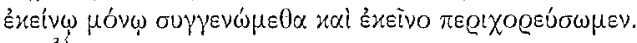

Cf. E. MOUTSOPoulos, «D'Aristote à Proclus : mouvement et clésir de l'Un dans la Théologie platonicienne, in id., Pbilosophie de la culture grecque, Athènes, Académie, 1998, p. 257-262, ò̀ il n'est cependant pas encore question de ce processus « musical». 
de ronde, par l'entité supérieure dont chaque entité choreliousa émane. L'acte de graviter suggère, dès lors, que l'entité désirante tente d'atteindre l'entité désirée en imitant son propre mouvement, donc en s'appropriant en l'occurrence ne serait-ce qu'une partie de ses qualités et de son comportement ${ }^{35}$. En réalité, graviter autour d'une entité supérieure revient, pour l'entité inférieure, à manifester sa propre participation au mouvement général de conversion. Ce qu'elle contient en elle de résidu de sa provenance de l'entité désirée lui interdit de s'en séparer et la rattache définitivement à elle. C'est pourquoi próodos et épistrophè s'avèrent définitivement liées l'une à l'autre et se complètent dans un état unitaire à deux volets. Progression et conversion ne sont pas deux étapes distinctes d'un mouvement qui se veut, tour à tour, de répulsion et d'attraction, comme dans le cas, par exemple, de la désagrégation et de la reconstitution des corps chez Empédocle ${ }^{36}$, étapes soumises, de surcrôt, à l'action distinctive d'un katros ${ }^{37}$ scandant les phases de leur alternance ${ }^{38}$; au contraire, elles se manifestent simultanément, d'où le caractère à la fois mouvementé et équilibré du système.

Enfin, troisièmement, il ressort que le jeu dialectique de la progression et de la conversion n'engendre pas un rapport en ligne directe entre les hypostases et les entités intermédiaires, mais suppose des mouvements circulaires des unes et des autres. En conséquence, il n'existe point de mouvements littéralement ascendants et descendants à l'intérieur du système. Si le système se présente en tant qu'ensemble structuré en mouvement, ce mouvement n'est pas effectué de haut en bas et vice-versa, mais de façon circulaire. On se trouverait ainsi face à un modèle qui fait appel à l'image du tourbillon. Il en découle que le système de Proclus, qui répond à des exigences à la fois ontologiques, épistémologiques et axiologiques ${ }^{39}$ se différencie du tout au tout du système de Plotin ${ }^{40}$. Il est calqué sur le modèle du chœur cyclique indéfiniment reproduit. Chaque entité est censée être choreute évoluant autour d'un centre occupé par l'entité immédiatement supérieure, et ainsi de suite.

35 Cf. supra et la note 27.

${ }^{36}$ Cf. E. Moutsopoulos, "Le modèle empédocléen de la pureté élémentaire et ses fonctions », in La cultura filosofica della Magna Grecia, Messina, GBM, 1989, p. 119-126.

${ }_{37}$ Cf. id., "Kairos et alternance : d’Empédocle à Platon», in o.c. (n. 33), p. 49-56.

38 Ainsi, dans l'ouvre de Teilhard de Chardin, on comparera le mouvement de remontée à partir de la lithosphère, et à travers la biosphère, puis de la noosphère, et qui s'achève au point oméga, à la phase de conversion platonicienne, mais qui suit en l'occurrence une phase de progression, ignorée ou mise entre parenthèses. $C f$. entre autre P. THEILARD DE CHARDIN, «L'histoire naturelle du monde » [1925], Euvres III, La vision du passé, Paris, Seuil, 1957, p. 143148.

39. En tant qu'» objet » de désir, l'entité désirée a tout d'une valeur (" centre d'intérêt »; " point d'attraction ») pour l'entité désirante. Cf. E. Moutsopoulos, Pbénoménologie des valeurs, Athènes, Éd. de l'Université, $1981^{2}$, p. 8 14; id., "Les valeurs : pérennité ou altérabilité ? ", Coexistence butmaine et développement durable, Montréal, Montmorency, 2001, p. 215-220.

40) C. i ., "Le modèle platonicien du système ontologique plotinien », Diotima 19 (1991), p. 9-12. 
C'est à l'intérieur de ce tourbillon complexe que se situe toute la vitalité du système dont on sait qu'il fut repris mutatis mutandis, pour être "christianisé », par le pseudo-Denys dans sa Hiérarchie céleste ${ }^{41}$, par les mystiques chrétienne, islamique et juive (kabale) médiévales, autant que par maintes philosophies de la Renaissance ${ }^{\text {iz }}$. Il est indéniable que le néoplatonisme proclusien, dont le caractère subtil aide à résoudre aisément les problèmes philosophiques autrement insolubles, a pu jouir d'une aussi large audience approbative en raison de sa structure, compliquée à outrance. Dieux et entités en plein remous ont hautement contribué à rendre le système proclusien fascinant autant que commode.

E. MOUTSOPOULOS

40, Hypsilantou

GR - 11521 ATHÈNES

"Cf. id., "La fonction catalytique de l'exaipbnès chez Denis, Diotima 23 (1994), p. 9-16.

${ }^{42}$ Cf. id., Les structures de l'imaginaire dans la philosophie de Proclus, Paris, Les Belles Lettres, 1985, p. 10-11. 